\title{
A Low Power IoT Network for Smart Agriculture
}

\author{
Soumil Heble, Ajay Kumar, K.V.V Durga Prasad, Soumya Samirana, P.Rajalakshmi \\ Department of Electrical Engineering \\ Indian Institute of Technology - Hyderabad, India \\ E-mail: \{soumilheble, ee15resch2002, ee15mtech11023, soumyasamirana, raji\}@iith.ac.in
}

\begin{abstract}
Traditional agriculture is transforming into smart agriculture due to the prominence of the Internet of Things (IoT). Low-cost and low-power are the key factors to make any IoT network useful and acceptable to the farmers. In this paper, we have proposed a low-power, low-cost IoT network for smart agriculture. For monitoring the soil moisture content, we have used an in-house developed sensor. In the proposed network, the IITH mote is used as a sink and sensor node which provides low-power communication. We have evaluated our network with state of the art networks, proposed for agriculture monitoring. Power and cost are the two metrics used for evaluation of these networks. Results show that the proposed network consumes less power and has prolonged lifetime in the agriculture field.
\end{abstract}

Index Terms-Smart Agriculture, Internet of Things, Soilmonitoring, Environmental-monitoring.

\section{INTRODUCTION}

Every field, from health to environment, education to entertainment and industry to home is embracing the Internet of Things (IoT) revolution [1]. Agriculture has seen many transformations and has adopted many machines to increase the yield. Field (soil and environmental parameters) and crop health monitoring are important factors for the yield to be of better quality and in larger quantities. In recent years, there have been many technological advancements in agriculture which have led to an increase in productivity and immunity of the crops. The technology which plays a key role in this is the Internet of Things (IoT) [2]. Traditional agriculture is transforming into smart agriculture due to the penetration of the Internet of Things (IoT) in the agricultural sector. The IoT networks are reducing human labor requirements by monitoring crop health and field environment remotely. IoT uses a wireless sensor network (WSN) as the backbone for gathering information for these monitoring and control applications. The hardware consists of end devices equipped with a variety of sensors to monitor various parameters like temperature, humidity, solar radiation, soil moisture, etc. and is capable of communicating this data to the other devices [3], [4]. About $70 \%$ of the fresh water available in the world is consumed by the agriculture sector [5], with the help of soil moisture sensor we can optimize the irrigation process and reduce the use of water [6], [7]. IoT is helping the farmers by monitoring growth stages of the crop, diseases, and estimation of the yield by giving otherwise restricted low-power, lowcost devices access to greater processing capabilities via the Internet.

Recently, for remote sensing, drones are widely being used for agriculture monitoring [8]. They are diminishing the role of satellites in monitoring and capturing images for agriculture by providing finer control and flexibility [9].

IoT systems for agriculture monitoring should be of lowcost such that it is affordable to the farmers and low-power for prolonged life of the network. In a typical monitoring network, there are many sensor nodes, a few sink nodes and a gateway depending on the network topology and area of the field. The sink collects data from the sensor nodes and uploads it to the server [10]. In most of the wireless networks, the sink is always in an active state and thus consumes a lot of power [11].

K. O. Flores et al. [12] have proposed a low-cost sensor system, they have used Xbee radios as sensor nodes for monitoring the agriculture parameters along with a Raspberry $\mathrm{Pi}$, which acts a gateway to the Internet. The authors in [13] proposed a three-layer architecture for precision agriculture wherein the processing of collected data happens on the server. It has a higher power consumption due to redundant transmissions. In the paper [14], for agriculture monitoring, Multi-mode, Multi-parameter, Multi-application Soil Sensing (M3SS) sensor node has been proposed. They have made the node capable of communicating in three wireless protocols namely WiFi, ZigBee, and Bluetooth which allows for greater flexibility in range, connectivity options and redundancy at the cost of higher power consumption.

These have motivated us to propose a low-power IoT network for smart agriculture. In this paper, we have proposed an IoT network based on the IITH mote [15] in which the sensor nodes are solar powered, which makes them self-sustaining by using an ambient energy source.

The rest of the paper is organized as follows: Section II discusses the proposed network architecture and its deployment. Section III evaluates our network for power consumption and cost analysis. The paper concludes in section IV with the future scope of work.

\section{Proposed Low Power Low Cost Iot Network}

We have considered an outdoor agriculture field of 648 square meters area, sown with maize crop. This field is divided into 27 plots, each spanning 24 square meters. In agriculture, the soil and environmental parameters both play an important role in crop health and yield. The network, nodes, and sink measure a total of 6 parameters. The soil moisture and soil temperature sensors are used for measuring the soil parameters. The environmental parameters measured include light intensity, relative humidity, and ambient temperature. The 
carbon dioxide and total solar radiation (TSR) sensors are interfaced to the sink. Each plot has one sensor node, totaling 27 nodes plus one sink in the maize field. Sensor nodes from each plot will send their data to the sink which will then upload it to a server for processing and consumption. The wireless hardware platform used for the sink and sensor node is the IITH mote (Fig. 1), an 802.15.4 compliant wireless mote which is developed in-house at the Wireless Networks (WiNet) lab, Indian Institute of Technology Hyderabad (IITH), India. We have designed a gateway to upload the data to the server and make it available online. An Intel Edison along with a $4 \mathrm{G}$ modem forms the gateway which communicates with an IITH mote acting as a sink (Fig. 2). The real-time hardware implementation of the network in the next section.

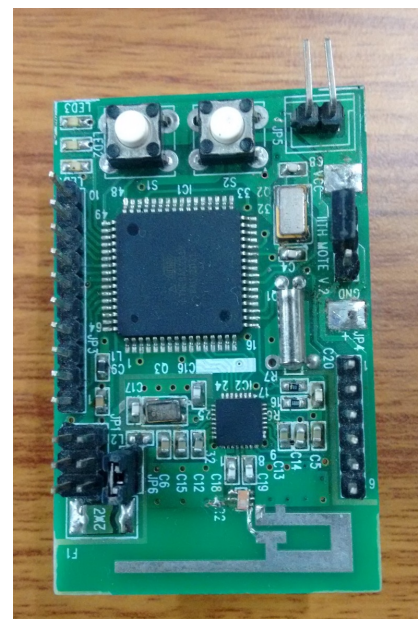

Fig. 1. IITH Mote

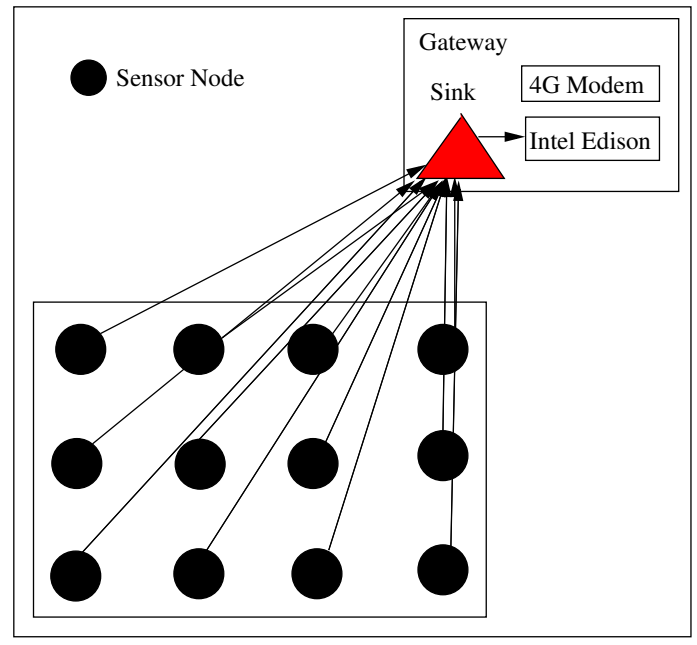

Fig. 2. Agricultural Monitoring Network Architecture

\section{A. Field Deployment}

1) Soil moisture sensor: Soil moisture is one of the most important factors for plant growth. Plants behave differently at different levels of soil water content. If the soil is dry, plants feel stressed, and the adverse effects of stress are reflected in their growth. Stress also makes them vulnerable to diseases and pests. The irrigation process can be optimized by precisely to meet the demands of the crop by observing the moisture level. Listed in descending order of cost and accuracy are some of the popular methods used to measure soil water content. Time domain reflectometry (TDR), frequency domain reflectometry (FDR), tensiometry, capacitance and resistance based methods. TDR sensors give most accurate results but are bulky and expensive. The resistance based technique is the cheapest method to measure soil moisture but, it offers poor accuracy and lifetime [16]. We invested our time in the design of a capacitance-based soil moisture sensor, Fig. , because the technology's ability to provides reasonable accuracy and lifetime for the cost.

The in-house designed sensor has a probe structure like an Inter-digital Transducer (IDT). It is a comb-like structure whose two electrodes form a capacitor. The electrodes of the transducer represent the plates of a capacitor, which are coplanar to each other allowing the electric field to fringe out of the transducer. This electric field then interacts with the measurand altering the dielectric in between the electrodes thereby altering the capacitance. The dielectric permittivity of air is about $\sim 1$, and that of soil is anywhere between $3-12$, which is small compared to dielectric permittivity of water which is about $\sim 80$. This huge difference is exploited by capacitance-based sensors to measure soil moisture. The probe is manufactured using standard PCB fabrication technology making it very cheap to mass-produce (Fig. 3. The probe's capacitance modulates the frequency of a square wave generator, which is measured by a high-speed timer circuit within a micro-controller. The frequency is then calibrated to soil moisture using gravimetric soil moisture measurement technique (Fig. 4).

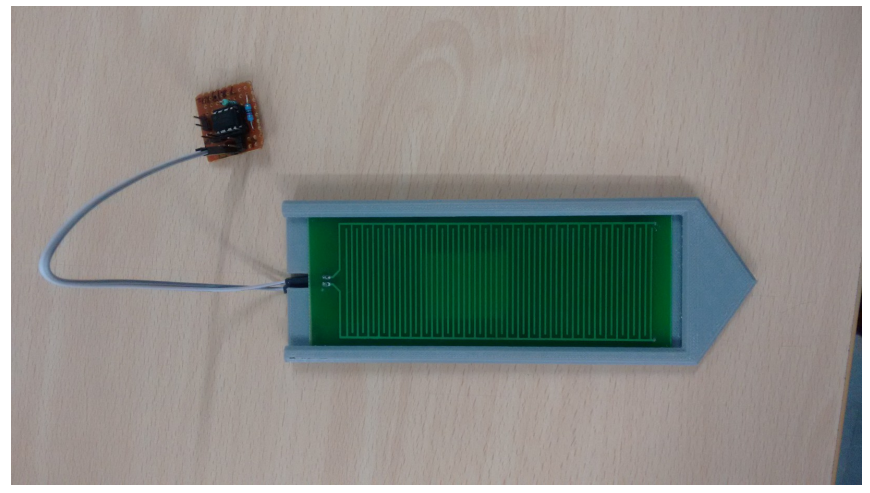

Fig. 3. Soil Moisture Sensor 


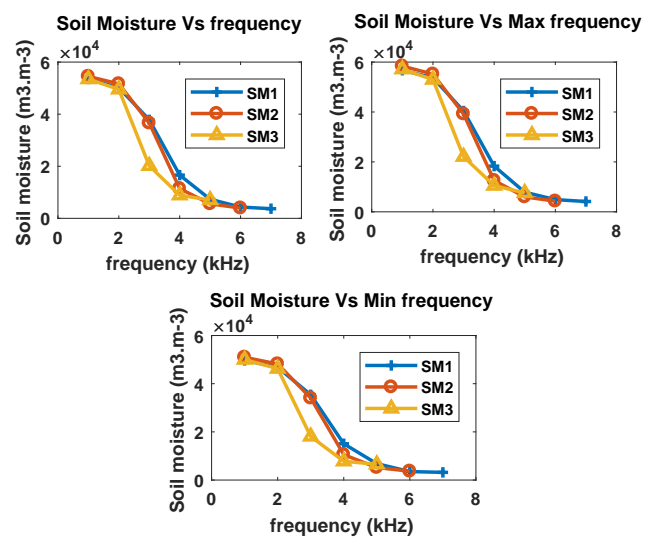

Fig. 4. Calibration and Repeatability Performance of the Sensor

2) Hardware deployment: The proposed network for agricultural monitoring is composed of spatially distributed nodes, each an IEEE 802.15.4 based wireless platform (IITH mote) with sensors and a solar based power circuit. The sensor nodes have a line of sight range of about 100 meters which means that the sensor node's transmission range covers the whole field and can easily reach the sink. The nodes sample their sensors hourly and send the data via the reliable unicast protocol to the sink and then go to the sleep state to conserve power. The sink is always on, but since every node uses the ContikiMAC RDC (radio duty cycling) to reduce its power consumption, it is a small price to pay for increased power saving. The sink transfers the collected data to an Intel Edison serially which then pushes the data to the server with the help of $4 \mathrm{G}$ modem. Since the maize crop field is in a remote location devoid of Internet hardline, we are using a 4G modem to connect the sink to the internet. The gateway is wall powered since the Intel Edison, and 4G Modem requires 7-12 $\mathrm{V}$ supply. The network is deployed in a maize crop field at the Professor Jayashankar Telangana State Agricultural University (PJTSAU), Hyderabad, India, Fig. 5 and Fig. 6.

Architecture II: The current requirement of the Intel Edison gateway could be a potential drawback for monitoring networks deployed at remote locations and agricultural fields, where laying electrical lines can be hazardous or impossible. To overcome this problem of running wires we have replaced the sink to internet link with a LoRa link which is a low-power, long-range modulation technique [17]. To emulate the gateway, we are using the Adafruit Feather 32u4 LoRa module, Fig. 7, as the LoRa gateway. It houses a LoRa transceiver based on Semtech's SX1276 LoRa modem running in the $868 \mathrm{MHz}$ ISM band (Fig. 8). After widespread deployment of LoRa, we will see mobile service providers commissioning their own highpower LoRa gateways on cellular towers and provide cheap service for upcoming LoRa end nodes.

\section{Evaluation of the Proposed Iot Network}

We have evaluated our proposed low-power, low-cost IoT Network by measuring power consumption and cost. In the

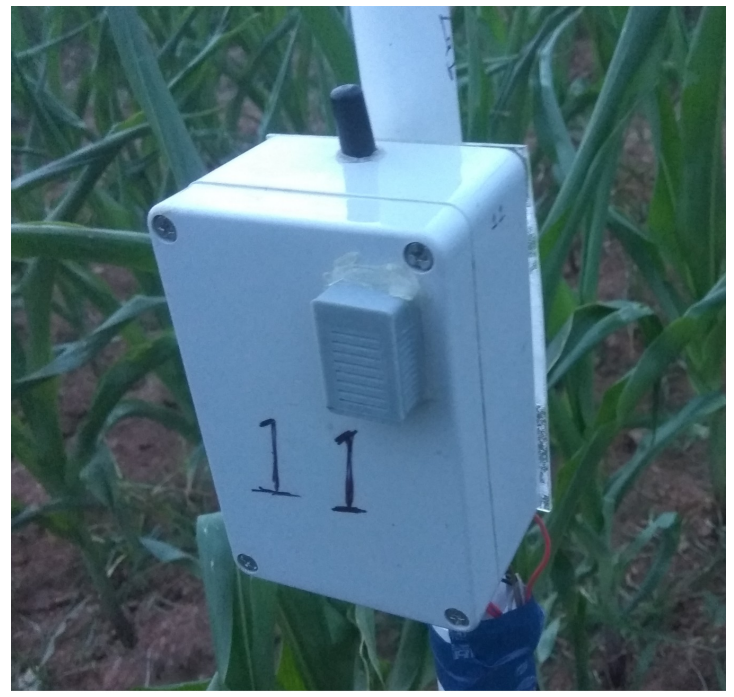

Fig. 5. Sensor Node Deployed in the Field

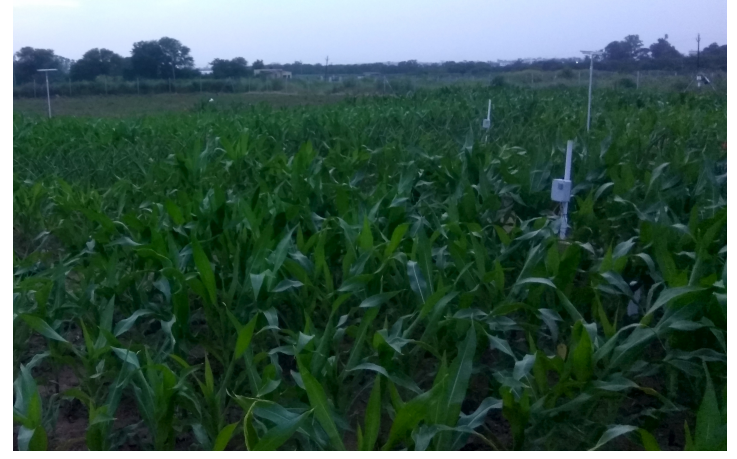

Fig. 6. Field Deployment of IoT Network in the Maize Crop Field

following section, we have compared the power consumption and cost of our proposed network with the already proposed networks in the literature.

\section{A. Power analysis}

For evaluating power consumption we have considered current consumption of different sensor nodes [19]-[25]. The current consumption of IITH mote (sensor node) and different sensor nodes is measured at different modes like sleep mode $I_{\text {sleep }}$, idle mode $I_{\text {idle }}$, transmit mode $I_{\text {transmission, }}$, and receive mode $I_{\text {receive }}$.

Our sensor node based on IITH mote consumes less power compared to the sensor node proposed in [14]. It consumes only $180 u A\left(I_{\text {sleep }}\right)$ current in sleep mode, $11.58 m A I_{\text {idle }}$ in idle mode, $26.58 \mathrm{~mA} I_{\text {transmission }}$ in transmit mode and $25.58 \mathrm{~mA} I_{\text {receive }}$ in receive mode, refer table I. From the table it is clear that the proposed network consumes less power compared to that of beforehand proposed networks.

1) Enhanced battery life: The solar panel is used to power and charges the battery of the sensor node, and it enhances the lifetime of the network by harvesting ambient energy. We have optimized the sleep time of sensor node to save power and prolong the lifetime of the network. In general, there will 


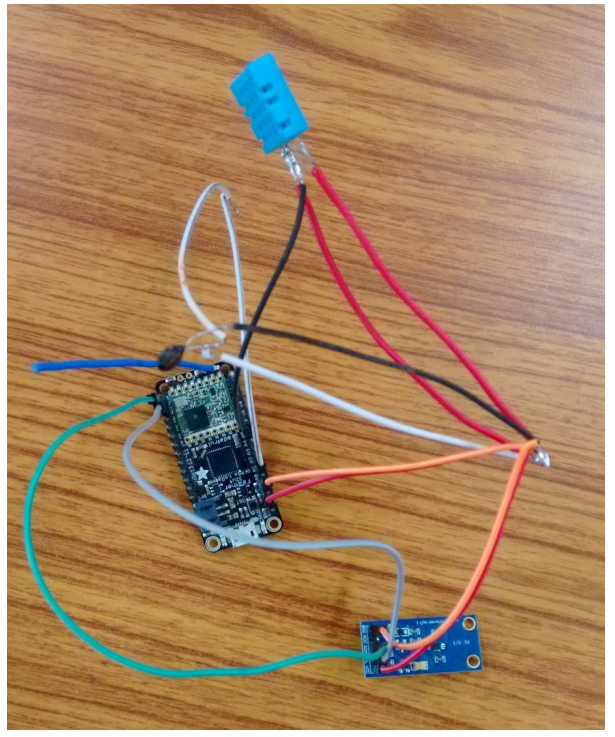

Fig. 7. LoRa Setup Prototype

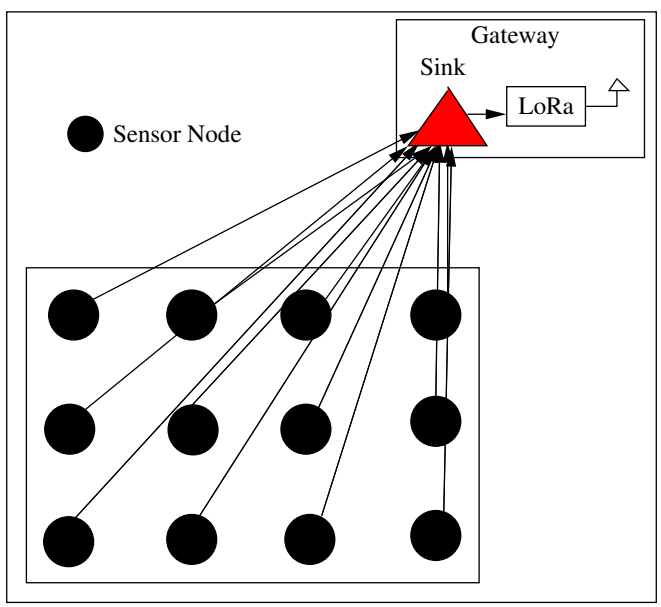

Fig. 8. Agricultural Monitoring Network Architecture II

be no rapid changes in agriculture parameters, hence the sensor nodes are sending the data every hour which saves power thereby enhancing the lifetime of the node in the field. The sensor node stays a maximum of $18 \mathrm{sec}, 373 \mathrm{msec}$ and 20 msec in idle, receive and transmit modes respectively. If sink acknowledges the data in the first transmission, then the end node can go to sleep earlier than the above mentioned time(s). We are using a $2000 \mathrm{mAh}$ lithium ion battery and considering a loss of $70 \%$ charge we have a realistic power budget of $1400 m A h$. Using ContikiMAC RDC and the sampling rate mentioned above we calculated a node lifetime of 243 days if used without solar power. Refer table II for the power consumption of the various sensors used.
TABLE I

\begin{tabular}{|c|c|c|c|}
\hline \multicolumn{4}{|c|}{ Power analysis of proposed low power IoT network } \\
\hline Sensor node & $I_{\text {sleep }}$ & $I_{\text {active }}$ & $I_{\text {transmission }}$ \\
\hline \hline IITH mote & $180 \mu \mathrm{A}$ & $11.58 \mathrm{~mA}$ & $26.58 \mathrm{~mA}$ \\
\hline M3SS & $200 \mathrm{~mA}$ & $500 \mathrm{~mA}$ & $600 \mathrm{~mA}$ \\
\hline DZ50 SPS & $3.3 \mu \mathrm{A}$ & $3.25 \mathrm{~mA}$ & $26.5 \mathrm{~mA}$ \\
\hline MicaZ SPS & $170 \mu \mathrm{A}$ & $4.35 \mathrm{~mA}$ & $18.5 \mathrm{~mA}$ \\
\hline TelosB PIS & $13 \mu \mathrm{A}$ & $1.72 \mathrm{~mA}$ & $19.12 \mathrm{~mA}$ \\
\hline ECO & $2 \mathrm{~mA}$ & $3 \mathrm{~mA}$ & $22 \mathrm{~mA}$ \\
\hline TinyNode & $5 \mu \mathrm{A}$ & $3 \mathrm{~mA}$ & $62 \mathrm{~mA}$ \\
\hline Waspmote & $62 \mu \mathrm{A}$ & $9 \mathrm{~mA}$ & $20 \mathrm{~mA}$ \\
\hline
\end{tabular}

TABLE II

\begin{tabular}{|c|c|c|c|}
\hline \multicolumn{4}{|c|}{ Power Analysis of Sensors Used } \\
\hline Sensor & Volts (V) & Current(mA) & Power $(\mathrm{mW})$ \\
\hline \hline DHT11 & 3.3 & 1.5 & 4.95 \\
\hline BH1750 & 3.3 & 0.2 & 0.66 \\
\hline Thermistor & 1.2 & 0.1 & 0.12 \\
\hline Soil Moisture & 3.3 & 1.4 & 4.62 \\
\hline Solar Radiation & 3.3 & 1 & 3.3 \\
\hline Carbon Dioxide & 5 & 60 & 300 \\
\hline
\end{tabular}

\section{B. Cost analysis}

The cost of the sensor and sensor node should be as low as possible for agriculture monitoring application. We have compared the cost of our sensor node with commercially available sensor nodes. The cost of the sensor node used in the proposed IoT network is $\$ 63$. From the table III, it can be observed that the cost of our mote is comparable to commercially available platforms [19]-[23] and already proposed sensor node [14]. Comparing with the waspmote [23] and [24], the cost of our sensor node is low. From the table, we can conclude that the proposed IoT network can be deployed at an affordable price for smart agriculture.

TABLE III

\begin{tabular}{|c|c|c|c|}
\hline \multicolumn{5}{|c|}{ Cost analysis of proposed low power IoT network } \\
\hline Sensor node & Processor unit & Radio unit & Cost \\
\hline \hline IITH mote & ATmega1281 & AT86RF230 & $63 \$$ \\
\hline M3SS & Intel SoC X1000 & CC2420/WiFi/BT2.0 & $53 \$$ \\
\hline SADmote & MSP430F1611 & MRF24J40 & $100 \$$ \\
\hline Tmote Sky & MSP430F1611 & CC2420 & $100 \$$ \\
\hline Waspmote & Atmega 1281 & Xbee & $200 \$$ \\
\hline ECO & 8051 controller & nRF24E1 & $57 \$$ \\
\hline TinyNode & TI MSP430 & SEMTECHCHSX1211 & $180 \$$ \\
\hline Waspmote & ATmega1281 & CC2420/Wi-Fi/BT4.0 & $147 \$$ \\
\hline
\end{tabular}

\section{CONCLUSION}

We have proposed and implemented a low-power IoT network for smart agriculture. We have used our in-house built IITH mote as a sink and sensor node. We have designed a low-cost soil moisture sensor. We have used soil moisture and soil temperature to measure soil parameter. The humidity, light intensity, ambient temperature sensors are used for measuring other environmental parameters. The proposed architecture is evaluated based on power consumption and cost. The 
evaluation results conclude that our network has a prolonged lifetime due to the optimized sleep time of sensor nodes and less power consumption by sensor nodes. The solar-powered feature of sensor nodes extends the lifetime of the network. In future, plan to use drone based remote monitoring for precision agriculture.

\section{REFERENCES}

[1] A. Al-Fuqaha, M. Guizani, M. Mohammadi, M. Aledhari and M. Ayyash, "Internet of Things: A Survey on Enabling Technologies, Protocols, and Applications," in IEEE Communications Surveys \& Tutorials, vol. 17, no. 4, pp. 2347-2376, Fourthquarter 2015.

[2] Ji-chun Zhao, Jun-feng Zhang, Yu Feng and Jian-xin Guo, "The study and application of the IOT technology in agriculture," 2010 3rd International Conference on Computer Science and Information Technology, Chengdu,pp. 462-465, 2010.

[3] Y. Liu, C. Zhang and P. Zhu, "The temperature humidity monitoring system of soil based on wireless sensor networks," 2011 International Conference on Electric Information and Control Engineering, Wuhan, pp. 1850-1853, 2011

[4] S. Ivanov, K. Bhargava and W. Donnelly, "Precision Farming: Sensor Analytics," in IEEE Intelligent Systems, vol. 30, no. 4, pp. 76-80, JulyAug. 2015.

[5] D. Pimentel, B. Berger, D. Filiberto, M. Newton, B. Wolfe, E. Karabinakis, S. Clark, E. Poon, E. Abbett, and S. Nandagopal, "Water resources: agricultural and environmental issues," BioScience, 54.10, 909-918, 2004.

[6] Kim, Yunseop, Robert G. Evans, and William M. Iversen, "Remote sensing and control of an irrigation system using a distributed wireless sensor network," IEEE transactions on instrumentation and measurement, 57.7 (2008): 1379-1387.

[7] J. Gutirrez, J. F. Villa-Medina, A. Nieto-Garibay and M. . PortaGndara, "Automated Irrigation System Using a Wireless Sensor Network and GPRS Module," in IEEE Transactions on Instrumentation and Measurement, vol. 63, no. 1, pp. 166-176, Jan. 2014.

[8] M. Ammad-udin, A. Mansour, D. Le Jeune, E. H. M. Aggoune and M. Ayaz, "UAV routing protocol for crop health management," 2016 24th European Signal Processing Conference (EUSIPCO), Budapest, pp. 1818-1822, 2016

[9] P. Lottes, R. Khanna, J. Pfeifer, R. Siegwart and C. Stachniss, "UAVbased crop and weed classification for smart farming," 2017 IEEE International Conference on Robotics and Automation (ICRA), pp. 30243031, 2017.

[10] I. F. Akyildiz, T. Melodia and K. R. Chowdury, "Wireless multimedia sensor networks: A survey," in IEEE Wireless Communications, vol. 14 no. 6, pp. 32-39, December 2007.

[11] C. F. Wang, J. D. Shih, B. H. Pan and T. Y. Wu, "A Network Lifetime Enhancement Method for Sink Relocation and Its Analysis in Wireless Sensor Networks," in IEEE Sensors Journal, vol. 14, no. 6, pp. 19321943, June 2014.

[12] K. O. Flores, I. M. Butaslac, J. E. M. Gonzales, S. M. G. Dumlao and R. S. J. Reyes, "Precision agriculture monitoring system using wireless sensor network and Raspberry Pi local server," 2016 IEEE Region 10 Conference (TENCON), Singapore, pp. 3018-3021, 2016.

[13] A. Khattab, A. Abdelgawad and K. Yelmarthi, "Design and implementation of a cloud-based IoT scheme for precision agriculture," 28th International Conference on Microelectronics (ICM), Giza, pp. 201-204, 2016.

[14] P. Kumar and S. R. N. Reddy, "Design and development of M3SS: A Soil Sensor Node for precision agriculture," 2016 International Conference on Recent Advances and Innovations in Engineering (ICRAIE), Jaipur, pp. 1-10, 2016.

[15] P.RajalakshmiIITH Mote-Wireless Sensor Communication Module, http://www.iith.ac.in/ raji/downloads/IITHmote-webpage.pdf.

[16] Huan, Zhan, Hui Wang, Chen Li, and Caiyan Wan, "The soil moisture sensor based on soil dielectric property," Personal and Ubiquitous Computing, vol. 21, no. 1, pp. 67-74, 2016.

[17] O. Georgiou and U. Raza, "Low Power Wide Area Network Analysis: Can LoRa Scale?," in IEEE Wireless Communications Letters, vol. 6, no. 2, pp. 162-165, April 2017.

[18] http://www.semtech.com/images/datasheet/sx1272.pdf
[19] Abdelraouf Ouadjaout, Noureddine Lasla, Miloud Bagaa, Messaoud Doudou, Cherif Zizoua, Mohamed Amine Kafi, Abdleouahid Derhab, Djamel Djenouri, Nadjib Badache, "DZ50: Energy-Efficient Wireless Sensor Mote Platform for Low Data Rate Applications," 5th International Conference on Emerging Ubiquitous Systems and Pervasive Networks (EUSPN, 2014.

[20] Atis Elsts, Rihards Balass, Janis Judvaitis, Reinholds Zviedris, Girts Strazdins, Artis Mednis, and Leo Selavo, "SADmote: A Robust and Cost-Effective Device for Environmental Monitoring," ARCS, LNCS, vol. 7179, pp. 225237, 2012.

[21] Chulsung Park, Jinfeng Liu, and Pai H. Chou, "Eco: an Ultra-Compact Low-Power Wireless Sensor Node for Real-Time Motion Monitoring", IPSN Fourth International Symposium on Information Processing in Sensor Networks, pp. 398-403, 2005.

[22] Mridula Maurya, Shri R. N. Shukla, "Current Wireless Sensor Nodes (Motes): Performance metrics and Constraints," International Journal of Advanced Research in Electronics and Communication Engineering (IJARECE), vol. 2, no. 1, 2013.

[23] https//www.cooking-hacks.com/shop/waspmote

[24] http//www.libelium.com/v11-files/documentation/waspmote/waspmotedatasheet 'eng.pdf

[25] http//www.libelium.com/downloads/documentation/4$20^{\circ}$ ma current loop' guide.pdf 